\title{
Toxoplasma gondii : comment un parasite amazonien est devenu un enjeu en matière de santé pour les Inuit
}

\author{
SJ Reiling ${ }^{1}$, BR Dixon ${ }^{1 *}$
}

\section{Résumé}

Toxoplasma gondii est un parasite protozoaire venant d'Amazonie. Les félidés (des mammifères de la famille des chats) constituent les seuls hôtes définitifs. Ces animaux perdent des ookystes infectieux en grand nombre dans l'environnement, ce qui par la suite peut infecter de nombreux hôtes intermédiaires comme des oiseaux, des mammifères et peut-être des poissons. La séroprévalence de l'infection à T. gondii chez l'humain est élevée dans certaines parties de l'Arctique canadien et on l'associe à des conséquences néfastes sur la santé parmi la population inuit. Étant donné que le territoire des félidés n'atteint pas l'Arctique, la façon à laquelle ce parasite s'est rendu en Arctique à partir de l'Amazonie ne saute pas aux yeux. Le présent aperçu a pour objectifs de résumer les répercussions de l'infection à T. gondii sur la santé des Inuit dans le Nord canadien, ainsi que de considérer la manière à laquelle l'infection a atteint le milieu nordique. Le présent article examine la prévalence de l'infection à T. gondii chez les animaux terrestres et marins de l'Arctique canadien et aborde leur rôle potentiel dans la transmission d'origine alimentaire de ce parasite à l'humain. Deux facteurs de distribution semblent plausibles. En premier lieu, les félidés des habitats plus au sud peuvent libérer des ookystes infectieux dans les cours d'eau. Puisque ces ookystes restent viables pendant plusieurs mois, ils peuvent être transportés vers le nord par le courant des rivières, fleuves et océans et infecter les poissons arctiques puis, au bout du compte, les mammifères marins qui se nourrissent de ces poissons. En second lieu, les hôtes intermédiaires terrestres et marins migrateurs peuvent transporter des kystes tissulaires de T. gondii dans l'Arctique, où ils peuvent ensuite transmettre l'infection aux carnivores. Le changement climatique fait en sorte que la migration des félidés vers le nord peut accroître la prévalence de l'infection à $T$. gondii au sein de la faune de l'Arctique. Les hôtes intermédiaires infectés chassés aux fins de subsistance accroissent le risque de transmission du parasite T. gondii à la population inuit, qui consomme souvent des aliments prélevés dans la nature, préparés selon les traditions.
Cette oeuvre est mise à la disposition selon les termes de la licence internationale Creative Commons Attribution 4.0

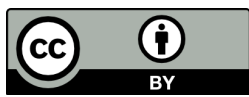

Affiliation

${ }^{1}$ Bureau des dangers microbiens, Direction des aliments, Santé Canada, Ottawa, Ontario

\section{${ }^{\star}$ Correspondance :}

brent.dixon@canada.ca

Citation proposée : Reiling SJ, Dixon BR. Toxoplasma gondii : comment un parasite amazonien est devenu un enjeu en matière de santé pour les Inuit. Relevé des maladies transmissibles au Canada 2019;45(7/8):203-10. https://doi.org/10.14745/ccdrv45i78a03f

Mots-clés : toxoplasmose, mammifères marins, poissons, changement climatique, oiseaux migrateurs

\section{Introduction}

\section{Infection à Toxoplasma gondii chez l'humain}

Toxoplasma gondii est un parasite protozoaire pouvant infecter presque l'ensemble des oiseaux et mammifères (1). Bien qu'au départ, ce parasite se soit développé dans la région de l'Amazonie de l'Amérique du Sud (2,3), on estime qu'il infecte maintenant deux milliards de personnes à travers le monde, avec un foyer de prévalence élevée en Amérique latine, en Europe orientale/centrale, au Moyen-Orient, en Asie du Sud-Est et en Afrique, de même qu'une prévalence moindre dans de nombreux pays européens, au Canada et aux États-Unis (4).
L'infection chez l'humain peut se faire par trois voies de transmission :

- Ingestion de kystes tissulaires lors de la consommation de la viande crue fraîche ou des organes crus frais d'un hôte intermédiaire infecté;

- Ingestion d'ookystes sporulés pouvant persister pendant plusieurs mois ou années dans le sol ou l'eau;

- Sur le plan congénital, de la mère au fœtus, lorsqu'une femme enceinte souffre de toxoplasmose aiguë (5). 
Lors de la phase d'infection initiale d'un hôte intermédiaire, y compris chez I'humain, l'infection à $T$. gondii se réplique rapidement et se propage dans les tissus, dont le cerveau (toxoplasmose aiguë). Chez l'humain, les symptômes peuvent être discrets et des personnes autrement en santé peuvent ne pas remarquer qu'elles sont infectées. Au bout du compte, la réplication parasitaire ralentit et les protozoaires se regroupent dans des kystes tissulaires (toxoplasmose latente). Les personnes atteintes de toxoplasmose latente qui deviennent immunocompromis peuvent développer une toxoplasmose réactivée, au cours de laquelle les parasites dormants dans les kystes tissulaires recommencent à se répliquer. Cette réactivation peut entraîner des symptômes semblables à la grippe graves, une vision trouble ou une encéphalite toxoplasmique. On associe également la toxoplasmose latente à des changements sur le plan des voies de signalisation cellulaire pouvant mener à des troubles neurologiques dont la schizophrénie, l'épilepsie, la maladie d'Alzheimer et la maladie de Parkinson (6-11). De plus, on a fait une association positive entre l'infection à T. gondii et le comportement de recherche du risque accru chez I'humain $(12,13)$. La transmission congénitale peut mener à la mortinaissance ou des complications neurologiques graves.

Les facteurs socioéconomiques peuvent avoir des conséquences importantes sur l'exposition humaine à ce parasite. Les facteurs qui influencent la séroprévalence chez l'humain comprennent la proximité des hôtes des réservoirs d'animaux domestiqués et sauvages, l'accès à l'eau potable, le style de vie urbain par rapport à rural, les types d'aliments consommés, la préparation des aliments (crus par rapport à la congélation, la cuisson et le séchage) et l'hygiène (se laver les mains et rincer les produits agricoles frais) (14).

\section{T. gondii de l'Amazonie à l'Arctique}

L'infection à Toxoplasma gondii s'est développée dans la forêt amazonienne $(2,3)$. Elle est très commune dans la région amazonienne et les populations autochtones du bassin de l'Amazone présentent le taux d'infection connu le plus élevé au monde : dans le cours supérieur de la rivière Negro, la séroprévalence de l'infection à T. gondii dépasse les 90 \% (15) Malgré sa distribution internationale, c'est seulement en Amazonie que l'infection à T. gondii se caractérise par un niveau élevé de diversité génétique et la présence de nombreux génotypes uniques (3). L'analyse du flux génétique des génotypes uniques indique qu'un petit nombre de lignées ancestrales a mené à la diversité actuelle de l'infection à $T$. gondii (2). L'hypothèse principale pour la propagation mondiale de l'infection à T. gondii est que le trafic maritime facilite le déplacement de chats domestiques et d'hôtes intermédiaires infectés vers d'autres continents (1). Le parasite se reproduit dans l'intestin grêle des félidés qui constituent des hôtes définitifs et des millions d'ookystes sont répandus dans l'environnement $(5,14)$. Le mode de propagation de l'infection à $T$. gondii de la forêt tropicale brésilienne à l'Arctique canadien est inconnu. Dans le présent article, les frontières de l'Arctique sont définies selon ce que décrit la Conservation de la flore et de la faune arctiques (CFFA), qui constitue le groupe de biodiversité du Conseil de l'Arctique. Le seul félidé sauvage qui habite dans le Nord canadien est le lynx du Canada, qui présente une séroprévalence de l'infection à T. gondii de $14 \%$ (16); cependant, le territoire du lynx ne s'étend pas au nord de la limite forestière (la forêt boréale ou la région subarctique). De plus, il y a peu de chats domestiques au sein des collectivités de l'Arctique canadien. Par conséquent, tandis que la présence de félidés infectés peut expliquer la propagation de l'infection à T. gondii dans la majeure partie de l'Amérique du Nord, elle n'explique pas la présence du parasite dans l'Arctique; de plus, malgré la rareté des félidés potentiellement infectés, l'infection à $T$. gondii reste présente dans un vaste éventail d'animaux de l'Arctique.

Pour compléter le cycle de vie du parasite, les ookystes répandus par les félidés qui constituent des hôtes définitifs doivent sporuler (figure 1) et être ingérés par les hôtes intermédiaires, qui constituent des proies potentielles pour les félidés et comprennent presque tous les animaux à sang chaud. L'infection à Toxoplasma gondii envahit les tissus de l'hôte intermédiaire et se répartit partout dans le corps, dont le cerveau (1). Cependant, les hôtes intermédiaires ne produisent pas d'ookystes; par conséquent, le mécanisme (ou les mécanismes) de dispersion géographique de l'infection à T. gondii, sans hôte définitif reste inconnu.

\section{Figure 1 : Cycle de vie de Toxoplasma gondii dans le Nord canadien}

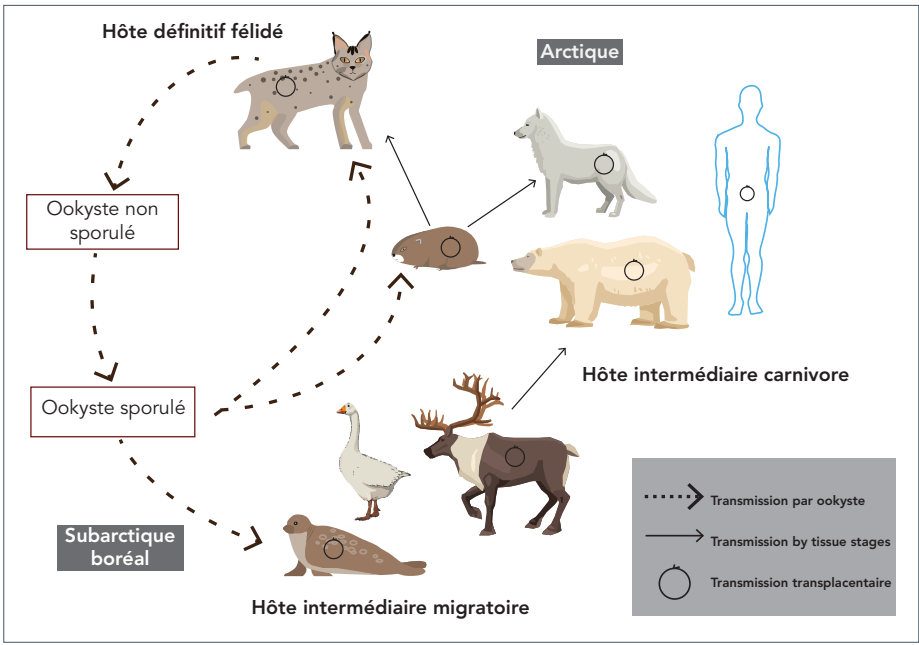

Cette image a été adaptée de : Jenkins EJ, Castrodale LJ, de Rosemond SJC, Dixon BR, Elmore SA, Gesy KM, Hoberg EP, Polley L, Schurer JM, Simard M, Thompson RCA. Tradition and Transition: Parasitic Zoonoses of People and Animals in Alaska, Northern Canada, and Greenland. Advances in Parasitology 2013;82:33-204. Reproduite avec la permission d'Elsevier

Le présent examen cherche à souligner l'incidence de ce parasite dans l'Arctique canadien et ses répercussions sur les populations inuit, ainsi qu'à considérer la manière à laquelle ce parasite est arrivé et est devenu endémique dans un milieu avec un manque d'hôtes définitifs. 


\section{Toxoplasma gondii dans l'Arctique}

\section{T. gondii chez l'Inuit du Canada}

Les infections à Toxoplasma gondii ont été signalées pour la première fois chez l'Inuit dans les années 1980 (17-19). Des études plus récentes indiquent que la séroprévalence de I'infection à T. gondii chez l'Inuit dans l'Arctique canadien varie de manière considérable selon la région (17). La séroprévalence de l'infection à Toxoplasma gondii chez l'adulte dans trois régions inuit canadiennes a été signalée à $8 \%$ au Nunatsiavut, $28 \%$ au Nunavut et $60 \%$ dans le Nunavik (20-24). Le nombre de données existantes ne suffit pas à déterminer si la prévalence de I'infection à T. gondii chez l'Inuit est stable ou a évolué au fil des décennies.

La nourriture traditionnelle a une grande importance culturelle pour les Inuit et est généralement considérée comme étant sûr et nutritif pour la majorité des gens. Par contre, il semble que $T$. gondii soit associée à la récolte et la consommation d'« aliments prélevés dans la nature », en particulier la viande et les organes, qu'on peut consommer crus $(19,25)$. La corrélation entre la séroprévalence de l'infection à $T$. gondii et les diverses pratiques de chasse et différentes habitudes alimentaires a fait l'objet d'un débat (26-29). Contrairement aux communautés inuit, les communautés cries voisines qui, en général, font cuire leur viande, présentent une séroprévalence de l'infection à $T$. gondii de seulement $5 \%$ (29). On a démontré que cuire la viande ou la faire congeler pendant plusieurs jours à des températures inférieures à zéro permet de tuer les agents pathogènes présents dans les kystes tissulaires (30).

Tandis que la toxoplasmose est souvent asymptomatique chez les personnes en santé, les femmes enceintes atteintes de toxoplasmose aiguë risquent de transmettre le parasite au fœtus en développement. En 1987, on a signalé une éclosion de toxoplasmose avec des femmes enceintes dans le Nunavik (19). L'infection était associée à l'écorchage des animaux et la consommation de viande de caribou crue (19).

\section{T. gondii sans hôte définitif}

Une étude de Svalbard, en Norvège, laisse entendre qu'on accorde trop d'importance au rôle des ookystes dans la transmission de l'infection à T. gondii aux animaux terrestres arctiques (31). L'archipel de Svalbard ne présente pas de chats domestiques ou sauvages, ce qui élimine la propagation d'ookystes de T. gondii infectieux dans l'environnement (31). L'absence d'ookystes de T. gondii à Svalbard est appuyée par des conclusions selon lesquelles les herbivores et oiseaux non migrateurs étaient séronégatifs pour l'infection à T. gondii (31). Cependant, les carnivores (renards) ont obtenu un résultat positif pour l'infection à T. gondii. Par conséquent, les oiseaux migrateurs pourraient avoir introduit le parasite T. gondii à Svalbard et, par la suite, les carnivores locaux sont devenus infectés en mangeant des proies infectées. Ainsi, il est possible pour l'infection à T. gondii d'être transmise d'un hôte intermédiaire à l'autre (p. ex. de l'oiseau au carnivore) sans reproduction sexuée du parasite chez un félidé qui constitue un hôte définitif. Ce cycle de transmission entre de nombreux hôtes intermédiaires peut expliquer la prévalence de l'infection à T. gondii dans l'Arctique, y compris l'Arctique canadien, surtout chez les carnivores autres que des félidés. Cette hypothèse est soutenue par des constatations selon lesquelles tous les oiseaux migrateurs et carnivores locaux de Svalbard analysés ont obtenu un résultat positif pour l'infection à T. gondii (31).

\section{Animaux terrestres de l'Arctique canadien}

Sans égard à la source de l'infection (ookystes environnementaux vs kystes tissulaires des proies infectées), de nombreux mammifères et oiseaux du Nord canadien ont obtenu un résultat positif pour l'infection à T. gondii (tableau 1). On a démontré que les oiseaux de partout au monde sont susceptibles de contracter une infection à T. gondii (31) et au Canada, les oiseaux migrateurs, tels que les oies, passent l'hiver dans des zones où les félidés sont communs et où il est possible de trouver des ookystes de T. gondii infectieux en grand nombre dans I'environnement (32-34). On a détecté l'infection à T. gondii dans les trois espèces d'oies analysées, avec la prévalence la plus élevée chez l'oie de Ross $(34,5 \%)$ et la plus faible chez la bernache du Canada (5,8 \%). Parmi les espèces de lagopèdes analysées, un seul lagopède alpin a obtenu un résultat positif pour l'infection à T. gondii, possiblement en raison de la faible exposition aux ookystes au sein de leurs habitats de la toundra arctiques, subarctiques et alpins.

\section{Tableau 1 : Oiseaux et mammifères terrestres analysés pour l'infection à Toxoplasma gondii dans l'Arctique canadien $^{\mathrm{a}}$}

\begin{tabular}{|c|c|c|c|c|}
\hline $\begin{array}{l}\text { Nom commun } \\
\text { (références) }\end{array}$ & Nom latin & $\begin{array}{c}\text { Nombre } \\
\text { analysé }\end{array}$ & $\begin{array}{l}\text { Nombre } \\
\text { de } \\
\text { résultats } \\
\text { positifs }\end{array}$ & $\begin{array}{c}\text { Pourcentage } \\
\text { de résultats } \\
\text { positifs }\end{array}$ \\
\hline \multicolumn{5}{|l|}{ Oiseaux } \\
\hline $\begin{array}{l}\text { Lagopède alpin } \\
\text { (35) }\end{array}$ & Lagopus muta & 25 & 1 & $4,0 \%$ \\
\hline $\begin{array}{l}\text { Lagopède des } \\
\text { saules (35) }\end{array}$ & $\begin{array}{l}\text { Lagopus } \\
\text { lagopus }\end{array}$ & 24 & 0 & $0,0 \%$ \\
\hline $\begin{array}{l}\text { Oie de Ross } \\
(36,37)\end{array}$ & Chen rossii & 357 & 123 & $34,5 \%$ \\
\hline $\begin{array}{l}\text { Petite oie des } \\
\text { neiges }(36,37)\end{array}$ & $\begin{array}{l}\text { Chen } \\
\text { caerulescens }\end{array}$ & 354 & 110 & $31,1 \%$ \\
\hline $\begin{array}{l}\text { Bernache du } \\
\text { Canada }(35,38)\end{array}$ & $\begin{array}{l}\text { Branta } \\
\text { canadensis }\end{array}$ & 240 & 14 & $5,8 \%$ \\
\hline \multicolumn{5}{|l|}{ Mammifères } \\
\hline \multicolumn{5}{|l|}{ Rongeurs } \\
\hline $\begin{array}{l}\text { Lemming } \\
\text { brun } \\
\text { néarctique } \\
\text { (37) }\end{array}$ & $\begin{array}{l}\text { Lemmus } \\
\text { trimucronatus }\end{array}$ & 84 & 0 & $0,0 \%$ \\
\hline \multicolumn{5}{|l|}{ Lagomorphes } \\
\hline $\begin{array}{l}\text { Lièvre } \\
\text { d'Amérique } \\
\text { (35) }\end{array}$ & $\begin{array}{l}\text { Lemmus } \\
\text { trimucronatus }\end{array}$ & 8 & 0 & $0,0 \%$ \\
\hline
\end{tabular}


Tableau 1 : Oiseaux et mammifères terrestres analysés pour l'infection à Toxoplasma gondii dans l'Arctique canadien $^{\mathrm{a}}$ (suite)

\begin{tabular}{|c|c|c|c|c|}
\hline $\begin{array}{l}\text { Nom commun } \\
\text { (références) }\end{array}$ & Nom latin & $\begin{array}{l}\text { Nombre } \\
\text { analysé }\end{array}$ & $\begin{array}{l}\text { Nombre } \\
\text { de } \\
\text { résultats } \\
\text { positifs }\end{array}$ & $\begin{array}{l}\text { Pourcentage } \\
\text { de résultats } \\
\text { positifs }\end{array}$ \\
\hline \multicolumn{5}{|l|}{ Mammifères } \\
\hline \multicolumn{5}{|c|}{ Lagomorphes (suite) } \\
\hline $\begin{array}{l}\text { Lièvre } \\
\text { arctique (35) }\end{array}$ & Lepus arcticus & 2 & 0 & $0,0 \%$ \\
\hline \multicolumn{5}{|l|}{ Ongulés } \\
\hline $\begin{array}{l}\text { Caribou de la } \\
\text { toundra (39) }\end{array}$ & $\begin{array}{l}\text { Rangifer } \\
\text { tarandus } \\
\text { groenlandicus }\end{array}$ & 117 & 43 & $36,8 \%$ \\
\hline Caribou (35) & $\begin{array}{l}\text { Rangifer } \\
\text { tarandus }\end{array}$ & 97 & 11 & $11,3 \%$ \\
\hline $\begin{array}{l}\text { Bœuf musqué } \\
(35,40)\end{array}$ & $\begin{array}{l}\text { Ovibus } \\
\text { moschatus }\end{array}$ & 348 & 16 & $4,6 \%$ \\
\hline \multicolumn{5}{|l|}{ Carnivores } \\
\hline $\begin{array}{l}\text { Renard } \\
\text { arctique (41) }\end{array}$ & $\begin{array}{l}\text { Vulpes } \\
\text { lagopus }\end{array}$ & 39 & 17 & $43,6 \%$ \\
\hline $\begin{array}{l}\text { Lynx du } \\
\text { Canada } \\
(16,35)\end{array}$ & $\begin{array}{l}\text { Lynx } \\
\text { canadensis }\end{array}$ & 173 & 44 & $25,4 \%$ \\
\hline Carcajou (42) & Gulo gulo & 41 & 17 & $41,5 \%$ \\
\hline Loup gris (35) & Canis lupus & 37 & 7 & $18,9 \%$ \\
\hline $\begin{array}{l}\text { Ours noir } \\
(35,43)\end{array}$ & $\begin{array}{l}\text { Ursus } \\
\text { americanus }\end{array}$ & 43 & 16 & $37,2 \%$ \\
\hline
\end{tabular}

Les rongeurs et lagomorphes arctiques canadiens ne présentaient pas de prévalence pour l'infection à $T$. gondii. Les lemmings bruns néarctiques ont obtenu un résultat négatif, tout comme les lièvres arctiques et les lièvres d'Amérique (tableau 1). La seule voie de transmission de l'infection à T. gondii pour les herbivores non migrateurs serait par l'ingestion de sol, de plantes ou d'eau contaminés par des ookystes infectieux. L'absence de prévalence de l'infection à T. gondii chez les rongeurs et lagomorphes de l'Arctique canadien appuie l'hypothèse selon laquelle les herbivores arctiques non migrateurs sont peu ou pas exposés aux ookystes de T. gondii infectieux (31).

L'exposition à l'infection à T. gondii des ongulés variait entre les espèces. Le caribou présente une prévalence de l'infection à T. gondii de $11,3 \%$, tandis que la sous-espèce caribou de la toundra présente une prévalence de $36,8 \%$. Il est difficile de comprendre pourquoi le caribou de la toundra présente une prévalence de l'infection à T. gondii si élevée. Le bœuf musqué présente une prévalence de l'infection à $T$. gondii de seulement $4,6 \%$ (tableau 1).

La prévalence de l'infection à $T$. gondii chez les carnivores était élevée dans toutes les espèces analysées, comme on pourrait s'y attendre même lorsque la prévalence du parasite dans leurs proies est relativement faible. Au Canada, on a constaté que la prévalence de l'infection à $T$. gondii était de $43,6 \%$ chez le renard arctique, $25,4 \%$ chez le lynx du Canada, $41,5 \%$ chez le carcajou, $18,9 \%$ chez le loup gris et $37,2 \%$ chez l'ours noir (tableau 1).

\section{Mammifères marins de l'Arctique canadien}

La plupart des pinnipèdes de l'Arctique canadien ont obtenu un résultat positif pour l'infection à $T$. gondii, y compris le phoque commun $(16,4 \%)$, le phoque annelé $(10,7 \%)$, le phoque barbu $(10,0 \%)$, le phoque à capuchon $(1,7 \%)$ et le morse $(14,7 \%)$ (tableau 2). On n'a pas détecté d'infection à Toxoplasma gondii chez le phoque du Groenland et d'autres études pourraient s'avérer utiles pour déterminer si des habitudes alimentaires différentes les protègent d'une exposition aux proies infectées.

Tableau 2 : Mammifères marins analysés pour l'infection à Toxoplasma gondii dans l'Arctique canadien ${ }^{a, b}$

\begin{tabular}{|c|c|c|c|c|}
\hline $\begin{array}{c}\text { Nom } \\
\text { commun } \\
\text { (références) }\end{array}$ & Nom latin & $\begin{array}{l}\text { Nombre } \\
\text { analysé }\end{array}$ & $\begin{array}{l}\text { Nombre } \\
\text { de } \\
\text { résultats } \\
\text { positifs }\end{array}$ & $\begin{array}{c}\text { Pourcentage } \\
\text { de résultats } \\
\text { positifs }\end{array}$ \\
\hline \multicolumn{5}{|l|}{ Pinnipeds } \\
\hline $\begin{array}{l}\text { Phoque } \\
\text { commun (26) }\end{array}$ & Phoca vitulina & 311 & 51 & $16,4 \%$ \\
\hline $\begin{array}{l}\text { Phoque } \\
\text { annelé }(26,35)\end{array}$ & Phoca hispida & 896 & 96 & $10,7 \%$ \\
\hline $\begin{array}{l}\text { Phoque du } \\
\text { Groenland } \\
(35,44)\end{array}$ & $\begin{array}{l}\text { Phoca } \\
\text { groenlandica }\end{array}$ & 113 & 0 & $0,0 \%$ \\
\hline $\begin{array}{l}\text { Phoque } \\
\text { barbu (26) }\end{array}$ & $\begin{array}{l}\text { Erignathus } \\
\text { barbatus }\end{array}$ & 20 & 2 & $10,0 \%$ \\
\hline $\begin{array}{l}\text { Phoque à } \\
\text { capuchon (44) }\end{array}$ & $\begin{array}{l}\text { Cystophora } \\
\text { cristata }\end{array}$ & 60 & 1 & $1,7 \%$ \\
\hline Morse (35) & $\begin{array}{l}\text { Odobenus } \\
\text { rosmarus }\end{array}$ & 34 & 5 & $14,7 \%$ \\
\hline \multicolumn{5}{|l|}{ Bears } \\
\hline $\begin{array}{l}\text { Ours blanc } \\
(35,44-47)\end{array}$ & Ursus maritimus & 599 & 67 & $11,2 \%$ \\
\hline \multicolumn{5}{|l|}{ Cetaceans } \\
\hline $\begin{array}{l}\text { Béluga } \\
(35,48)\end{array}$ & $\begin{array}{l}\text { Delphinapterus } \\
\text { leucas }\end{array}$ & 69 & 13 & $18,8 \%$ \\
\hline $\begin{array}{l}\text { Baleine } \\
\text { boréale (35) }\end{array}$ & $\begin{array}{l}\text { Balaena } \\
\text { mysticetus }\end{array}$ & 2 & 1 & $50,0 \%$ \\
\hline
\end{tabular}

Comprend les animaux arctiques saisonniers

${ }^{b}$ Comprend le golfe Amundsen, le golfe du Saint-Laurent, la baie d'Hudson, la mer du Labrador et la mer de Beaufort

Les ours blancs constituent les seuls ursidés considérés comme des mammifères marins, puisqu'ils dépendent de l'océan pour la nourriture et l'habitat. On a détecté l'infection à Toxoplasma gondii chez l'ours blanc dans la partie continentale canadienne et la mer de Beaufort, avec une prévalence globale de $11,2 \%$.

On a analysé deux espèces de cétacés arctiques pour l'infection à T. gondii : le béluga et la baleine boréale (tableau 2). La prévalence de l'infection à Toxoplasma gondii chez les bélugas 
de l'ouest de l'Arctique canadien était de 18,8 \% (tableau 2). Parmi les deux baleines boréales analysées, un animal a obtenu un résultat positif pour l'infection à T. gondii (35).

\section{T. gondii dans les eaux de l'Arctique}

On a détecté I'ADN du parasite Toxoplasma gondii dans jusqu'à $77 \%$ des échantillons d'eau de surface et de puits traitée et non traitée à l'échelle mondiale $(49,50)$. Dans certaines régions du Canada, on a associé la hausse des précipitations aux nombres élevés d'ookystes de T. gondii dans l'eau de surface (51). La plupart des rivières et fleuves canadiens coulent vers le nord; $39 \%$ de l'eau douce du pays se jette dans la baie d'Hudson et $36 \%$ dans l'océan Arctique (52). Les ookystes emportés dans l'eau de mer peuvent rester infectieux pendant deux ans et se répandre à l'aide des courants océaniques (20,53-55).

On a avancé I'hypothèse selon laquelle les poissons pourraient constituer le chaînon manquant entre les ookystes qui se retrouvent dans les bassins versants et l'infection chez les mammifères marins (56). On a trouvé des ookystes de Toxoplasma gondii dans le tube digestif d'un poisson sauvage (57) et on a prouvé que les ookystes peuvent rester infectieux dans le tube digestif d'un poisson pendant plusieurs heures (58), fournissant de ce fait une source d'infection possible pour les superprédateurs. Jusqu'à maintenant, on a uniquement signalé une infection expérimentale de poissons avec des kystes tissulaires de T. gondii chez le poisson zèbre et uniquement dans des conditions strictement contrôlées (57). On a également signalé une infection à Toxoplasma gondii dans une gamme de mollusques et crustacés à l'échelle mondiale (59), ce qui pourrait fournir une autre source d'infection chez les mammifères marins et l'humain, bien que ce point n'ait pas encore été consigné ni confirmé dans l'Arctique.

Afin de déterminer si les poissons de l'Arctique constituent une source potentielle de T. gondii, nous avons analysé les tissus musculaires de 121 poissons d'eau douce et euryhalins du Nunavik pour déceler la présence d'ADN du parasite T. gondii. Quinze poissons (12,4\%) ont obtenu un résultat positif pour T. gondii au moyen de la réaction en chaîne de la polymérase pour l'amplification de l'ADN, suivie du séquençage de Sanger. Le saumon atlantique et l'omble chevalier présentent une prévalence de l'infection à T. gondii de $26,7 \%$ et $12,0 \%$, respectivement. Les autres espèces de poissons ayant obtenu un résultat positif pour l'ADN du parasite $T$. gondii sont le touladi $(2,9 \%)$ et l'omble de fontaine (16,7\%). On a détecté I'infection à Toxoplasma gondii dans un chabot $(n=1)$, mais pas chez le brochet ou le grand corégone, possiblement en raison de la petite taille de l'échantillon ( $n=2$ et 6 , respectivement) (Reiling, S.J., R. Boone, H. Merks et B.R. Dixon. Données non publiées, 2018). Il s'agit de conclusions préliminaires et un plus grand nombre de poissons de l'Arctique canadien sont en cours d'analyse dans notre laboratoire pour détecter la présence de T. gondii.

\section{Discussion}

Il existe de nombreux mécanismes par lesquels le parasite T. gondii peut avoir été introduit dans l'Arctique canadien. Le parasite Toxoplasma gondii pourrait avoir été introduit par des oiseaux et mammifères migrateurs qui ont contracté l'infection lors de l'ingestion d'ookystes (qui peuvent persister dans le sol et l'eau des régions géographiques où l'on trouve des félidés) ou de proies infectées, dans leurs habitats au sud, pour ensuite transporter l'infection avec eux vers le nord. Le parasite pourrait ensuite être transmis d'un hôte intermédiaire à l'autre dans l'Arctique, et ce, même sans hôte définitif. De plus, les prédateurs tels que le renard arctique, le carcajou et le loup gris présentent une prévalence de l'infection à T. gondii élevée, ce qui laisse entendre que le carnivorisme pourrait également constituer une importante voie de transmission dans l'Arctique. Les ookystes répandus par les félidés dans le sud et transportés vers le nord au moyen des cours d'eau pourraient constituer une autre source d'infection des animaux aquatiques dans l'Arctique. Jusqu'à tout récemment, on ignorait que les poissons constituaient une source potentielle d'infection à T. gondii. Cependant, nos conclusions préliminaires laissent entendre qu'on peut trouver l'infection à T. gondii dans les poissons de l'Arctique canadien et qu'ils pourraient constituer une autre source d'infection chez les humains et les mammifères piscivores.

Les facteurs environnementaux qui accroissent la prévalence de I'infection à T. gondii chez les animaux chassés par les Inuit aux fins de subsistance peuvent représenter une menace croissante pour la santé des Inuit dans les régions arctiques du Canada. D'autres études sont nécessaires pour déterminer l'influence des changements environnementaux et socioéconomiques sur la prévalence de l'infection à T. gondii chez les animaux et les humains de l'Arctique canadien.

Le changement climatique et la hausse des températures peuvent favoriser la croissance forestière dans des régions qui, auparavant, étaient trop froides (60-62). L'accroissement du couvert forestier pourrait élargir l'habitat des félidés sauvages, augmentant de ce fait la perte d'ookystes de T. gondii dans l'environnement (20). Les nombres élevés d'ookystes combinés au réchauffement des températures pourraient accroître le potentiel d'infection des hôtes intermédiaires, y compris les oiseaux et mammifères dont on ignore encore qu'ils constituent des hôtes du parasite T. gondii dans l'Arctique canadien. Cette situation pourrait ensuite ouvrir de nouvelles voies de transmission chez les humains qui consomment des aliments prélevés dans la nature préparés selon les traditions.

\section{Conclusion}

La toxoplasmose est maintenant répandue dans une grande partie de l'Amérique du Nord et du Sud, principalement par l'entremise de félidés. Malgré l'absence de félidés dans ce territoire, T. gondii est maintenant présent dans l'Arctique Canadien et pose un risque de santé aux Inuit, principalement 
aux femmes enceintes et aux personnes immunodéprimées. La source d'infection à T. gondii la plus probable chez les Inuit est via les hôtes intermédiaires infectés et la consommation de mets préparés de manière traditionnelle, la viande et les abats pouvant être consommés crus. La prévention des infections par la cuisson et la congélation complète du poisson, de la viande et des abats ainsi qu'une meilleure compréhension des modes de transmissions des zoonoses aideront à réduire ce risque.

\section{Déclaration des auteurs}

S. J. R. a recueilli et analysé les données. S. J. R. et B. R. D. ont rédigé, révisé et approuvé le manuscrit.

\section{Conflit d'intérêts}

Aucun.

\section{Remerciements}

Nous souhaitons remercier A. Iqbal et S. Lamhoujeb d'avoir fourni l'ADN des poissons. R. Boone et $\mathrm{H}$. Merks ont fourni une excellente assistance technique.

\section{Financement}

Le présent travail a reçu l'appui du Conseil de recherches en sciences naturelles et en génie (CRSNG) du Canada, du Programme de bourses de recherche scientifique dans les laboratoires du gouvernement canadien (S. J. R.) et de Santé Canada (B. R. D.).

\section{Références}

1. Dubey JP. General Biology. In: Toxoplasmosis of Animals and Humans (Second Ed.). Boca Raton, London, New York: Taylor and Francis Group; 2009. pp. 1-20. ISBN 978-1-4200-9237-0 2010. CRC Press.

2. Su C, Khan A, Zhou P, Majumdar D, Ajzenberg D, Dardé ML, Zhu XQ, Ajioka JW, Rosenthal BM, Dubey JP, Sibley LD. Globally diverse Toxoplasma gondii isolates comprise six major clades originating from a small number of distinct ancestral lineages. Proc Natl Acad Sci USA 2012;109(15):5844-9. DOI

3. Shwab EK, Zhu XQ, Majumdar D, Pena HF, Gennari SM, Dubey JP, Su C. Geographical patterns of Toxoplasma gondii genetic diversity revealed by multilocus PCR-RFLP genotyping. Parasitol. 2014;141(4):453-61. DOI

4. Pappas G, Roussos N, Falagas ME. Toxoplasmosis snapshots: global status of Toxoplasma gondii seroprevalence and implications for pregnancy and congenital toxoplasmosis. Int J Parasitol 2009;39(12):1385-94. DOl
5. Jones JL, Dubey JP. Waterborne toxoplasmosis-recent developments. Exp Parasitol 2010;124(1):10-25. DOI

6. Ngô HM, Zhou Y, Lorenzi H, Wang K, Kim TK, Zhou Y, Bissati KE, Mui E, Fraczek L, Rajagopala SV, Roberts CW, Henriquez FL, Montpetit A, Blackwell JM, Jamieson SE, Wheeler K, Begeman IJ, Naranjo-Galvis C, Alliey-Rodriguez N, Davis RG, Soroceanu L, Cobbs C, Steindler DA, Boyer K, Noble AG, Swisher CN, Heydemann PT, Rabiah P, Withers S, Soteropoulos P, Hood L, McLeod R. Toxoplasma modulates signature pathways of human epilepsy, neurodegeneration \& cancer. Sci Rep 2017;7(1):11496. DOI

7. Burgdorf KS, Trabjerg BB, Pedersen MG, Nissen J, Banasik K, Pedersen OB, Sørensen E, Nielsen KR, Larsen MH, Erikstrup C, Bruun-Rasmussen P, Westergaard D, Thørner LW, Hjalgrim $H$, Paarup HM, Brunak S, Pedersen CB, Torrey EF, Werge T, Mortensen PB, Yolken RH, Ullum H. Large-scale study of Toxoplasma and Cytomegalovirus shows an association between infection and serious psychiatric disorders. Brain Behav Immun. 2019: pii: S0889-1591(18)30699-8. DOI

8. Fabiani S, Pinto B, Bonuccelli U, Bruschi F. Neurobiological studies on the relationship between toxoplasmosis and neuropsychiatric diseases. J Neurol Sci 2015;351(1-2):3-8. Epub 2015 Feb 21. DOI

9. Sugden K, Moffitt TE, Pinto L, Poulton R, Williams BS, Caspi A. Is Toxoplasma gondii infection related to brain and behavior impairments in humans? Evidence from a population-representative birth cohort. PLoS One 2016;11(2):e0148435. DOI

10. Flegr J, Horáček J. Toxoplasmosis, but not borreliosis, is associated with psychiatric disorders and symptoms. Schizophr Res. 2018;197:603-4. DOI

11. Hamdani N, Bengoufa D, Godin O, Doukhan R, Le Guen E, Daban-Huard C, Bennabi M, Delavest M, Lépine JP, Boukouaci W, Laouamri H, Houenou J, Jamain S, Richard JR, Lecorvosier P, Yolken R, Rajagopal K, Leboyer M, Tamouza R. Immunoglobulin sub-class distribution in bipolar disorder and schizophrenia: potential relationship with latent Toxoplasma gondii infection. BMC Psychiatry 2018;18(1):239. $\mathrm{DOI}$

12. Sutterland $A L$, Kuin $A$, Kuiper $B$, van Gool $T$, Leboyer $M$, Fond $G$, de Haan L. Driving us mad: the association of Toxoplasma gondii with suicide attempts and traffic accidents - a systematic review and meta-analysis [Epub ahead of print]. Psychol Med 2019;49(9):1-16. DOI

13. Samojłowicz D, Twarowska-Małczyńska J, Borowska-Solonynko A, Poniatowski ŁA, Sharma N, Olczak M. Presence of Toxoplasma gondii infection in brain as a potential cause of risky behavior: a report of 102 autopsy cases. Eur J Clin Microbiol Infect Dis 2019;38(2):305-17. Epub 2018 Nov 23. DOI

14. Dubey JP, Frenkel JK. Cyst-induced toxoplasmosis in cats. J Protozool 1972;19(1):155-77. DOI PubMed

15. Bóia MN, Carvalho-Costa FA, Sodré FC, Pinto GM, Amendoeira MR. Seroprevalence of Toxoplasma gondii infection among indian people living in lauareté, Sao Gabriel da Cachoeira, Amazonas, Brazil. Rev Inst Med Trop São Paulo 2008;50(1):17-20. DOI PubMed 
16. Simon A, Bigras Poulin M, Rousseau AN, Dubey JP, Ogden $\mathrm{NH}$. Spatiotemporal dynamics of Toxoplasma gondii infection in Canadian lynx (Lynx canadensis) in western Québec, Canada. J Wildl Dis 2013;49(1):39-48. DOI

17. Tanner CE, Staudt M, Adamowski R, Lussier M, Bertrand S, Prichard RK. Seroepidemiological study for five different zoonotic parasites in northern Quebec. Can J Public Health 1987;78(4):262-6. PubMed

18. Curtis MA, Rau ME, Tanner CE, Prichard RK, Faubert GM, Olpinski S, Trudeau C. Parasitic zoonoses in relation to fish and wildlife harvesting by Inuit communities in northern Quebec, Canada. Arctic Med Res 1988;47 Suppl 1:693-6. PubMed

19. McDonald JC, Gyorkos TW, Alberton B, MacLean JD, Richer $G$, Juranek D. An outbreak of toxoplasmosis in pregnant women in northern Québec. J Infect Dis 1990;161(4):769-74. $\mathrm{DOI}$

20. Jenkins EJ, Castrodale LJ, de Rosemond SJ, Dixon BR, Elmore SA, Gesy KM, Hoberg EP, Polley L, Schurer JM, Simard M, Thompson RC. Tradition and transition: parasitic zoonoses of people and animals in Alaska, northern Canada, and Greenland. Adv Parasitol 2013;82:33-204. DOI

21. Messier V, Lévesque $B$, Proulx JF, Rochette L, Libman MD, Ward BJ, Serhir B, Couillard M, Ogden NH, Dewailly E, Hubert B, Déry S, Barthe C, Murphy D, Dixon B. Seroprevalence of Toxoplasma gondii among Nunavik Inuit (Canada). Zoonoses Public Health 2009;56(4):188-97. DOI

22. Egeland GM. Inuit Health Survey 2007-2008: Nunavut. Centre for Indigenous Peoples' Nutrition and Environment, 2010. www.mcgill.ca/cine/files/cine/adult_report_nunavut. pdf

23. Egeland GM. Inuit Health Survey 2007-2008: Nunatsiavut. Centre for Indigenous Peoples' Nutrition and Environment, 2010. www.mcgill.ca/cine/files/cine/adult_report_-_ nunatsiavut.pdf

24. Goyette S, Cao Z, Libman M, Ndao M, Ward BJ. Seroprevalence of parasitic zoonoses and their relationship with social factors among the Canadian Inuit in Arctic regions. Diagn Microbiol Infect Dis 2014;78(4):404-10. DOI

25. Parkinson AJ. The Arctic Human Health Initiative: a legacy of the International Polar Year 2007-2009. Int J Circumpolar Health 2013;72(1):21655. DOI

26. Statistiques Canada. Enquête auprès des peuples de 2006: Santé, éducation et nourriture traditionnelle chez les Inuits. Ottawa (ON) : Stats Can; date de modification : 2013. https://www150.statcan.gc.ca/n1/pub/89-637-x/2008004/art/ art1-fra.htm

27. Simon A, Chambellant M, Ward BJ, Simard M, Proulx JF, Levesque B, Bisgras-Poulin M, Rousseau AN, Ogden NH. Spatio-temporal variations and age effect on Toxoplasma gondii seroprevalence in seals from the Canadian Arctic. Parasitology 2011;138(11):1362-8. DOI
28. Lévesque B, Messier V, Bonnier-Viger Y, Couillard M, Côté S, Ward BJ, Libman MD, Gingras S, Dick D, Dewailly E. Seroprevalence of zoonoses in a Cree community (Canada). Diagn Microbiol Infect Dis 2007;59(3):283-6. DOI

29. Campagna $S$, Lévesque $B$, Anassour Laouan-Sidi E, Côté S, Serhir B, Ward BJ, Libman M, Drebot MA, Makowski K, Andonova M, Ndao M, Dewailly E. Seroprevalence of 10 zoonotic infections in 2 Canadian Cree communities. Diagn Microbiol Infect Dis 2011;70(2):191-9. DOI

30. El-Nawawi FA, Tawfik MA, Shaapan RM. Methods for inactivation of Toxoplasma gondii cysts in meat and tissues of experimentally infected sheep. Foodborne Pathog Dis 2008;5(5):687-90. DOI

31. Prestrud KW, Asbakk K, Fuglei E, Mork T, Stien A, Ropstad E, Tryland M, Gabrielsen GW, Lydersen C, Kovacs KM, Loonen MJ, Sagerup K, Oksanen A. Serosurvey for Toxoplasma gondii in arctic foxes and possible sources of infection in the high Arctic of Svalbard. Vet Parasitol 2007;150(1-2):6-12. DOl

32. VanWormer E, Fritz H, Shapiro K, Mazet JA, Conrad PA. Molecules to modeling: toxoplasma gondii oocysts at the human-animal-environment interface. Comp Immunol Microbiol Infect Dis 2013;36(3):217-31. DOI

33. Gotteland C, McFerrin BM, Zhao X, Gilot-Fromont E, Lélu M. Agricultural landscape and spatial distribution of Toxoplasma gondii in rural environment: an agent-based model. Int J Health Geogr 2014;13(1):45. DOI

34. Shwab EK, Saraf $P$, Zhu XQ, Zhou DH, McFerrin BM, Ajzenberg D, Schares G, Hammond-Aryee K, van Helden P, Higgins SA, Gerhold RW, Rosenthal BM, Zhao X, Dubey JP, Su C. Human impact on the diversity and virulence of the ubiquitous zoonotic parasite Toxoplasma gondii. Proc Natl Acad Sci USA 2018;115(29):E6956-63. DOl

35. Al-Adhami BH, Simard M, Hernández-Ortiz A, Boireau C, Gajadhar AA. Development and evaluation of a modified agglutination test for diagnosis of Toxoplasma infection using tachyzoites cultivated in cell culture. Food and Waterborne Parasitology. 2016;2:15-21. DOI

36. Elmore SA, Huyvaert KP, Bailey LL, Milhous J, Alisauskas RT, Gajadhar AA, Jenkins EJ. Toxoplasma gondii exposure in arctic-nesting geese: A multi-state occupancy framework and comparison of serological assays. Int J Parasitol Parasites Wildl 2014;3(2):147-53. DOI

37. Elmore SA, Samelius G, Fernando C, Alisauskas RT, Jenkins EJ. Evidence for Toxoplasma gondii in migratory vs. nonmigratory herbivores in a terrestrial arctic ecosystem. Can J Zool 2015;93(8):671-5. DOI

38. Verma SK, Calero-Bernal R, Cerqueira-Cézar CK, Kwok OC, Dudley M, Jiang T, Su C, Hill D, Dubey JP. Toxoplasmosis in geese and detection of two new atypical Toxoplasma gondii strains from naturally infected Canada geese (Branta canadensis). Parasitol Res 2016;115(5):1767-72. DOI 
39. Kutz SJ, Elkin BT, Panayi D, Dubey JP. Prevalence of Toxoplasma gondii antibodies in barren-ground caribou (Rangifer tarandus groenlandicus) from the Canadian Arctic. J Parasitol 2001;87(2):439-42. DOI

40. Kutz SJ, Elkin B, Gunn A, Dubey JP. Prevalence of Toxoplasma gondii antibodies in muskox (Ovibos moschatus) sera from northern Canada. J Parasitol 2000;86(4):879-82. PubMed

41. Elmore SA, Samelius G, Al-Adhami B, Huyvaert KP, Bailey LL, Alisauskas RT, Gajadhar AA, Jenkins EJ. Estimating Toxoplasma gondii exposure in Arctic foxes (Vulpes lagopus) while navigating the imperfect world of wildlife serology. J Wildl Dis 2016;52(1):47-56. DOI

42. Reichard MV, Torretti L, Garvon JM, Dubey JP. Prevalence of antibodies to Toxoplasma gondii in wolverines from Nunavut, Canada. J Parasitol 2008;94(3):764-5. DOI

43. Philippa JD, Leighton FA, Daoust PY, Nielsen O, Pagliarulo M, Schwantje H, Shury T, Van Herwijnen R, Martina BE, Kuiken T, Van de Bildt MW, Osterhaus AD. Antibodies to selected pathogens in free-ranging terrestrial carnivores and marine mammals in Canada. Vet Rec 2004;155(5):135-40. DOI PubMed

44. Measures LN, Dubey JP, Labelle P, Martineau D. Seroprevalence of Toxoplasma gondii in Canadian pinnipeds. J Wildl Dis 2004;40(2):294-300. DOl

45. Atwood TC, Duncan C, Patyk KA, Nol P, Rhyan J, McCollum M, McKinney MA, Ramey AM, Cerqueira-Cézar CK, Kwok OC, Dubey JP, Hennager S. Environmental and behavioral changes may influence the exposure of an Arctic apex predator to pathogens and contaminants. Sci Rep 2017;7(1):13193. DOI

46. Kirk CM, Amstrup S, Swor R, Holcomb D, O'Hara TM. Morbillivirus and Toxoplasma exposure and association with hematological parameters for southern Beaufort Sea polar bears: potential response to infectious agents in a sentinel species. EcoHealth 2010;7(3):321-31. DOl

47. Rah H, Chomel BB, Kasten RW, Hew CH, Farver TB, Follmann EH, Garner GW, Amstrup SC. Serosurvey of selected zoonotic agents in polar bears (Ursus maritimus). Vet Rec 2005;156(1):7-13. DOI

48. Haman KH, Raverty S, Wendte JM, Loseto LL, Ferguson SH, Grigg ME. Infected tissues from hunter harvested beluga (Delphinapterus leucas) in the Western Canadian Arctic. 44th Annual IAAAM Conference; 2013; The Marine Mammal Center Sausalito, California.

49. Bahia-Oliveira L, Gomez-Marin J, Shapiro K. Toxoplasma gondii. In: J.B. Rose and B. Jiménez-Cisneros, editors. Global Water Pathogen Project. Michigan State University, E. Lansing, MI, UNESCO. 2017. http://www.waterpathogens. org/book/toxoplasma-gondii

50. Shapiro K, Conrad PA, Mazet JA, Wallender WW, Miller WA, Largier JL. Effect of estuarine wetland degradation on transport of Toxoplasma gondii surrogates from land to sea. Appl Environ Microbiol 2010 Oct;76(20):6821-8. DOI
51. Bowie WR, King AS, Werker DH, Isaac-Renton JL, Bell A, Eng SB, Marion SA. Outbreak of toxoplasmosis associated with municipal drinking water. The BC Toxoplasma Investigation Team. Lancet 1997 Jul;350(9072):173-7. DOI

52. Statistique Canada. L activité humaine et I environnement. Section 2 : L'offre d'eau au Canada-réserves et débits. Ottawa (ON): Stats Can 2010. https://www150.statcan.gc.ca/ n1/pub/16-201-x/2010000/part-partie2-fra.htm

53. Lindsay DS, Dubey JP. Long-term survival of Toxoplasma gondii sporulated oocysts in seawater. J Parasitol 2009;95(4):1019-20. DOI

54. Gómez-Marin JE, de-la-Torre A, Angel-Muller E, Rubio J, Arenas J, Osorio E, Nuñez L, Pinzon L, Mendez-Cordoba LC, Bustos A, de-la-Hoz I, Silva P, Beltran M, Chacon L, Marrugo M, Manjarres C, Baquero H, Lora F, Torres E, Zuluaga OE, Estrada M, Moscote L, Silva MT, Rivera R, Molina A, Najera S, Sanabria A, Ramirez ML, Alarcon C, Restrepo N, Falla A, Rodriguez T, Castaño G. First Colombian multicentric newborn screening for congenital toxoplasmosis. PLoS Negl Trop Dis 2011;5(5):e1195. DOI

55. Afonso E, Thulliez P, Gilot-Fromont E. Local meteorological conditions, dynamics of seroconversion to Toxoplasma gondii in cats (Felis catus) and oocyst burden in a rural environment. Epidemiol Infect 2010;138(8):1105-13. DOI

56. Simon A, Poulin MB, Rousseau AN, Ogden NH. Fate and transport of Toxoplasma gondii oocysts in seasonally snow covered watersheds: a conceptual framework from a melting snowpack to the Canadian arctic coasts. Int J Environ Res Public Health 2013;10(3):994-1005. DOI

57. Sanders JL, Zhou Y, Moulton HM, Moulton ZX, McLeod R, Dubey JP, Weiss LM, Kent ML. The zebrafish, Danio rerio, as a model for Toxoplasma gondii: an initial description of infection in fish. J Fish Dis 2015;38(7):675-9. DOI

58. Massie GN, Ware MW, Villegas EN, Black MW. Uptake and transmission of Toxoplasma gondii oocysts by migratory, filter-feeding fish. Vet Parasitol 2010;169(3-4):296-303. DOI

59. Shapiro K, Bahia-Oliveira L, Dixon B, Dumètre A, de Wit LA, VanWormer E, Villena I. Environmental transmission of Toxoplasma gondii: oocysts in water, soil and food. Food and Waterborne Parasitology. 2019;15:e00049. DOI

60. Lenoir J, Gégout JC, Marquet PA, de Ruffray P, Brisse H. A significant upward shift in plant species optimum elevation during the 20th century. Science 2008;320(5884):1768-71. $\mathrm{DOI}$

61. Feeley KJ, Silman MR, Bush MB, Farfan W, Cabrera KG, Malhi Y, Meir P, Salinas Revilla N, Natividad Raurau Quisiqupanqui M, Saatchi S. Upslope migration of Andean trees. J Biogeogr 2011;38(4):783-91. DOI

62. Fei S, Desprez JM, Potter KM, Jo I, Knott JA, Oswalt CM. Divergence of species responses to climate change. Sci Adv 2017;3(5):e1603055. DOI 